\title{
Fuzzy Reference Gain-Scheduling Approach as Intelligent Agents: FRGS Agent
}

\author{
J. E. ARAUJO * \\ ernestolit.inpe.br \\ * Integration and Testing Laboratory - LIT \\ Computer Sci. and Applied Math. Associated Lab - LAC \\ Instituto Nacional de Pesquisas Espaciais - INPE \\ Av. Astronautas, 1758 \\ 12.227-010 - São José dos Campos (SP) - BRAZIL
}

\author{
S. A. SANDRI \\ sandra@lac.inpe.br
}

\# Department of Electronics Engineering

Instituto Tecnológico de Aeronáutica - ITA

Praça Marechal Eduardo Gomes, 50

São José dos Campos (SP) - BRAZIL

\begin{abstract}
Goal driven Intelligent Agents and Fuzzy Reference Gain-Scheduling (FRGS) approach are described in this paper as interchangeable concepts that are able to deal with dynamic complex problems. It is advocated that the FRGS approach may be viewed as an autonomous goal-based agent, that is, a fuzzy logic based agent able to autonomously adapt itself to environmental changes introduced by external inputs. The concept of fuzzy systems and intelligent agent are employed in decision-making problems to lead to a certain degree of autonomy in decision support system. Although the FRGS method was originally proposed for control application, this approach was extended to decision-making tasks due to its ability of emulating human reasoning. This new agent approach uses the external input information also denominated reference (goal) as the driven mechanism to determine the behavior of the system in order to achieve the desired objectives (goal). Thus, the FRGS approach can be modeled in the framework of an adaptive and goal (also context or environment) driven agent.
\end{abstract}

\section{INTRODUCTION}

The concept of intelligent agent has been employed along with fuzzy sets in decision-making problems to lead to a certain degree of autonomy in decision support systems [12][15][19]. Relevant application areas for this concept are planning, scheduling, and control problems, where one can collect data, monitor processes and make local intelligent decisions, as well as recommendations [17][18].

The fuzzy theory proposed by Zadeh to deal with fuzzy sets and afterwards with fuzzy logic has been proving its effectiveness and showing its value in many fields of research. Although the future of fuzzy approach keeps flattering, it is also not easy to be predicted. Nevertheless, it could be realized that in the present time a new frontier for this research area is coming up in which fuzzy theory is being associated with intelligent agents to build new sorts of autonomous systems [1][6][11][13].

Intelligent agents can be thought as decision-making units that are capable of getting information (perception or measurement), reasoning about what must be done (judgment and conclusion) and acting upon the external environment to reach a desired task (control functions). Although there are different interpretations about what an agent is, there is a consensus that they have to possess special skills, such as, being autonomous (i.e., independent), reactive (i.e., responding to events), pro-active (i.e., initiating actions of their own volition), and social (i.e., communicative). Sometimes stronger features are added (beliefs, desired, intentions) giving intention notions for agents.

Despite the fact that an agent exhibits the interesting ability to represent intelligent behavior, one issue that comes up is how the agents represent their intelligence since intelligent systems typically require some form of knowledge representation and the knowledge has to come from somewhere 0. An approach for knowledge based systems is the rule-based system for representing and processing knowledge in terms of rules, that is, a knowledge base in the form of IF-THEN rules, a database, and an inference mechanism for reasoning [9]. A special form of knowledge base system, able of reasoning by using rules in an environment with uncertainty, is the fuzzy systems. This approach incorporates the expertise of humans and allows the approximation of human reasoning. Since a fuzzy system emulates human reasoning, it can be applied in planning, scheduling, and control problems. This approach makes use of a set of membership functions and a set of rules for the purpose of representing the knowledge.

As mentioned, an agent must be able to perceive the environment, make decision, represent sensed data, acquire knowledge, infer rules, and, finally, interact with the environment. When an agent interacts with the environment it may act directly upon the world as any control system or indirectly influences another mechanism to act upon the world. This definition of an agent is so far almost identical with that of control systems as any controller must sense a system to be controlled, represent the data, manipulate the information and determine an action to modify part of an environment or universe. Thus, in this paper, control systems and agents are considered to be interchangeable concepts. Additionally, the Fuzzy Reference Gain-Scheduling (FRGS) approach and goal-based Intelligent Agents (IA) are shown also as interchangeable concepts, which are able to deal with decision making in complex problems. In so doing that the 
former one will supply a framework for adaptive behavior for the latter one. The FRGS approach is formally presented in [4] and it was applied as an efficient set of autonomous actions to supervise and maintain safe an industrial system [3]. Although it has been presented as a synthesis to control complex problems it can also be employed in decisionmaking tasks [2]. The FRGS methodology introduces adaptive decision and control performance into autonomous systems when there are changes in the objective or goal (reference) to be achieved. Extended to intelligent agents, this approach allows adaptive behavior according to goals, intentions, desires, or beliefs. In so doing, the FRGS approach may be modeled as a particular class of goal driven agents that employ fuzzy logic rule-based systems.

\section{FUZzY CONTROLLER, FUZZY DECISION AND INTELLIGENT AGENT}

Like a control system, an agent may be understood as if it perceives its environment through sensors and act upon that environment through effectors (actuators). Nevertheless, while an intelligent agent is the one that employs reasoning to find out the appropriate action to solve a problem, it is not every control approach that uses reasoning to find out the action to control a system. The control approach that incorporates reasoning is the fuzzy controller. In a glimpse, fuzzy control theory and intelligent agents can be understood as presenting similar behavior since they have the same properties (TABLE 1).

Another correspondence between agents and fuzzy controllers is that the former one is itself a static nonlinear mapping between its inputs and outputs. It maps input to output through coding (fuzzification) inference mechanism and decoding (defuzzification) processes. Like fuzzy control systems, intelligent agents are programs that map a possible percept sequence into appropriate actions according to an objective to be achieved.

Fuzzy control systems are based on the fuzzy theory, which uses the concept of fuzzy sets associate with the vague and imprecise information, the compositional rule of inference that is used to reason (make inference) and to make decisions associated with the fuzzy logic. The inference mechanism is based on a set of rules of the kind IF <condition> THEN <conclusion> that contains information about the relationships between the inputs and outputs.

One advantage of this approach is that fuzzy controllers represent and manipulate information in the same way human beings reason when dealing with uncertain or vague information. Further, if a learning mechanism is adopted, fuzzy control systems permit the adjustment of the membership functions related to linguistic variables as well as the adjustment of the set of conditional-action rules. This characteristic allows them to learn from a set of input and output and behave on its own. Artificial neural-network, genetic algorithm, simulated annealing are some examples of learning mechanisms applied to tune fuzzy control systems [10][14]. Thus, being able of emulating the human reasoning process fuzzy controllers may also be considered to be independent as presenting a certain degree of autonomy.

In [9] and [2], it is shown that by using the compositional rule of inference, it is possible to treat the fuzzy control approach and the decision-making process similarly.

Fuzzy decision-making and fuzzy control together can also be found in the literature [10][17][20][22]. In addition, fuzzy decision-making and fuzzy control process can also be viewed into the context of agent theory in order to make decisions. Fuzzy decision applied together with fuzzy control and agents are presented in [18][21].

In order to carry on the selection process of deciding which the appropriate action is, an agent must satisfy a

TABLE 1

SIMILARITIES BETWEEN FUZZY CONTROL AND INTELLIGENT AGENT

\begin{tabular}{|c|c|c|c|}
\hline Characteristics & $\begin{array}{c}\text { Fuzzy } \\
\text { Control } \\
\end{array}$ & $\begin{array}{c}\text { Intelligent } \\
\text { agent }\end{array}$ & $\begin{array}{l}\text { FRGS } \\
\text { Control }\end{array}$ \\
\hline $\begin{array}{l}\text { sense the environment to decide } \\
\text { about what should be the } \\
\text { appropriate action to change de } \\
\text { environment; }\end{array}$ & $X$ & $\mathrm{X}$ & $\mathrm{X}$ \\
\hline $\begin{array}{l}\text { act upon the environment by } \\
\text { using effectors (actuator); }\end{array}$ & $\mathrm{X}$ & $\mathrm{X}$ & $\mathrm{X}$ \\
\hline $\begin{array}{l}\text { map the dates from input to } \\
\text { output; }\end{array}$ & $\mathrm{X}$ & $\mathrm{X}$ & $\mathrm{X}$ \\
\hline $\begin{array}{ll}\begin{array}{l}\text { decide upon } \\
\text { requirements } \\
\text { constraints etc.) }\end{array} & \begin{array}{l}\text { equivalent } \\
\text { (objective, }\end{array} \\
\end{array}$ & $\mathrm{X}$ & $\mathrm{X}$ & $\mathrm{X}$ \\
\hline $\begin{array}{l}\text { are rational since they decide } \\
\text { about what to do by using } \\
\text { reasoning - in this case a set of } \\
I F-T H E N \text { rules; }\end{array}$ & $X$ & $\mathrm{X}$ & $\mathrm{X}$ \\
\hline $\begin{array}{l}\text { are reactive since they perceive } \\
\text { the environment, decide, and } \\
\text { respond to changes that occur in } \\
\text { it; }\end{array}$ & $\mathrm{X}$ & $\mathrm{X}$ & $\mathrm{X}$ \\
\hline $\begin{array}{l}\text { are pro-active because fuzzy } \\
\text { control systems are able to decide } \\
\text { based on reference (goal- } \\
\text { directed); }\end{array}$ & - & $X$ & $\mathrm{X}$ \\
\hline $\begin{array}{l}\text { are autonomous, when associated } \\
\text { with any mechanism of } \\
\text { optimization to adjust its } \\
\text { parameters and keep control over } \\
\text { their actions and internal state, } \\
\text { independently of the human } \\
\text { being; }\end{array}$ & $\mathrm{X}$ & $\mathrm{X}$ & $\mathrm{X}$ \\
\hline $\begin{array}{l}\text { present social ability in the case } \\
\text { of being inserted into distributed } \\
\text { artificial intelligence or } \\
\text { distributed control systems or } \\
\text { when in cooperation with } \\
\text { humans, or other agents in order } \\
\text { to achieve their tasks. }\end{array}$ & $\mathrm{X}$ & $\mathrm{X}$ & $X$ \\
\hline
\end{tabular}


performance index (measure) or goal. In a fuzzy environment, the decision-making process uses the concept of fuzzy goal, fuzzy restriction and fuzzy decision in such a way that the goal and restriction are fuzzy sets in the same space of alternative [5]. This sort of decision-making process may be studied from different perspectives; however, all of them have in common the advantage that fuzzy goals may be derived from a given performance index. Intelligent agents and fuzzy theory applied together to make decision may be found in the literature [1][11][12][15][17][19].

In the context exposed here, all those characteristics make the fuzzy control approach, as well as the decision-making process, and intelligent agent interchangeable concepts that are able to deal with decision-making. Since the reasoning employed by fuzzy systems are based on a set of IF-THEN rules (also called situation-action rules), the structure of this agent can be classified as a reflexive agent [16]. Another characteristic present in both agent and fuzzy control system is the ability to represent the internal state. The internal state representation is guaranteed because fuzzy control systems can deal with present and past information about the environment to be manipulated (for instance, error and error variation). Thus, from this perspective, a fuzzy controller is also a reflexive agent with internal state.

\section{FRGS AGENTS: A NEW FRAMEWORK FOR INTELLIGENT AGENT}

The drawback of traditional fuzzy decision approach is that there is no adaptation, when there are changes in the objective (goal) to be achieved. Objective here may also be interpreted as desires, intentions, plan (goals), or beliefs, in the belief-desire-intention (BDI) agent paradigm [7] of the class of procedural reasoning system (PRS) agent architecture. One way to suppress this disadvantage and introduce adaptive performance into the system is to use the Fuzzy Reference Gain Scheduling (FRGS) approach applied both in control systems as well as in decision-making processes. That is possible because of the inherent ability of FRGS systems to change their membership functions (parameters) according to different operational conditions represented by the goals, or any exogenous parameter. This approach may change its condition or conclusion as well as the rules in accordance with changes in external inputs.

\section{A. FRGS Control Approach}

As it was mentioned during the introduction, the fuzzy reference gain scheduling approach has formally been presented in [3] and [4] as an adaptive control approach.

The adaptive mechanism is carried out by the selection of several operating points determined by external reference state trajectories that modify the support and core of the membership functions of fuzzy controllers (Fig. 1 and Fig. 2).
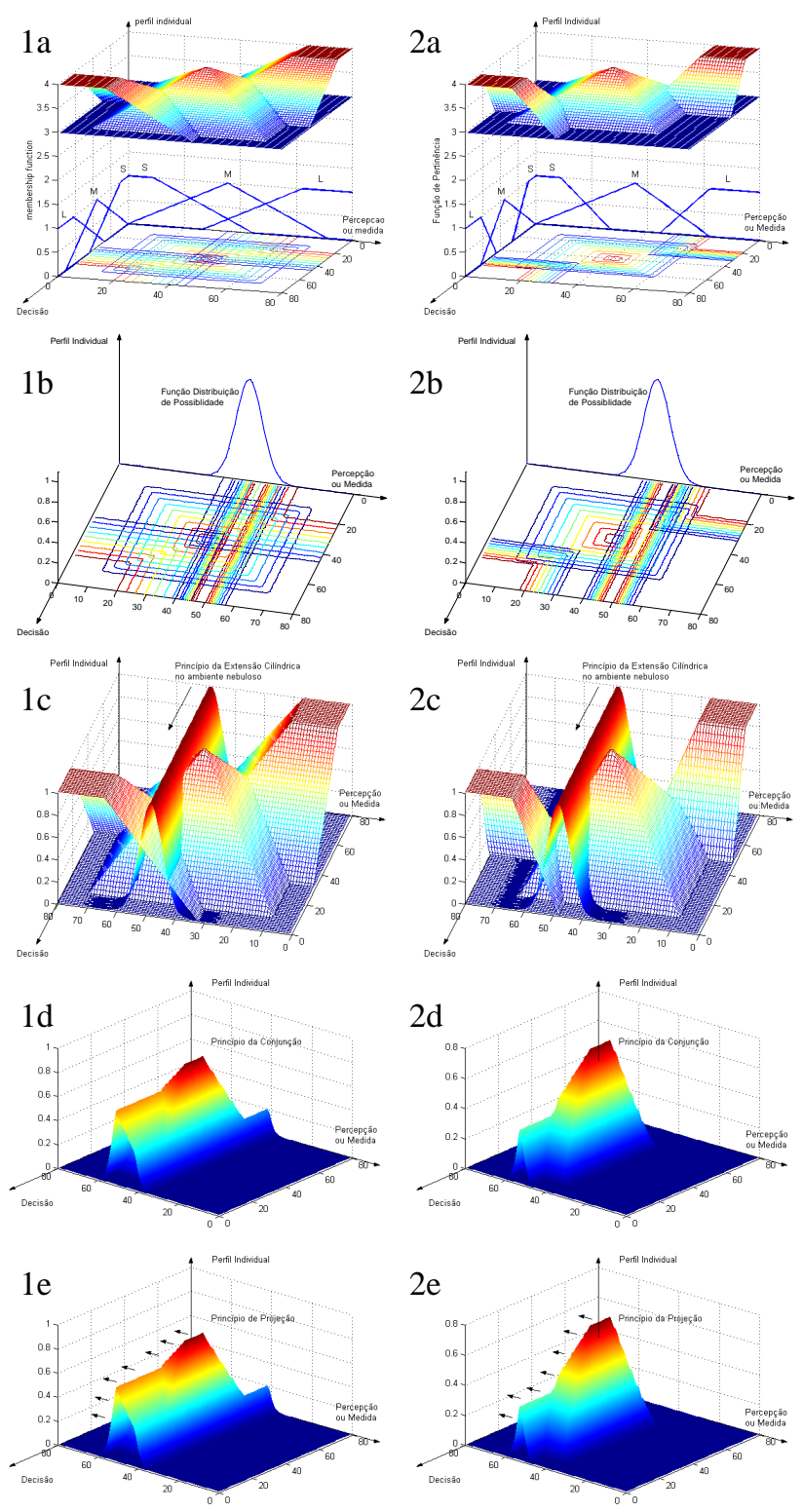

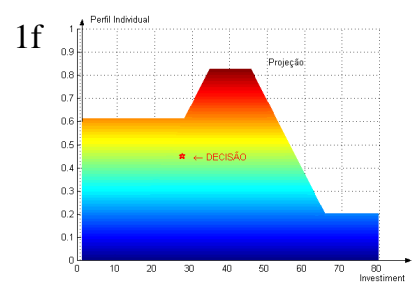

Fig. 1 - Example 1
Fig. 2 - Example 2

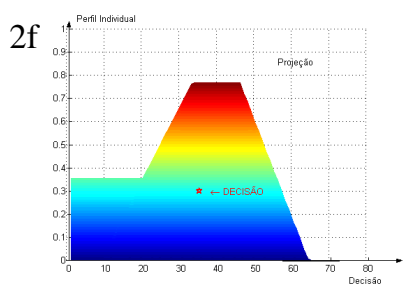

\section{Membership functions and fired area of control action.}

In so doing, the control surfaces will change as required by operational conditions, determined by the reference. Different from the scaling factor method, the FRGS method permits the mixing of constant and adaptive fuzzy sets (linguistic terms) on-line. It permits parameters to change homogeneously, as a scaling factor, or allows some parameters to modify 
independently or even stay constant. This adaptive mechanism may be used in the coding (fuzzification) or in the decoding (defuzzification) processes, as well as in the $I F$ THEN rules, and with different inferences schemes (e.g. Mamdani, Sugeno, Tsukamoto etc.).

Like any other fuzzy controller, it incorporates the expertise of human beings acquired in past experiences to figure out an approach to control/supervise those processes that, for instance, are not linear and whose dynamics change with time according to operational conditions, and/or present time-delay. Due to its ability to deal with human reasoning, FRGS can be extended to decision-making task [2].

\section{B. FRGS Decision Approach}

The use of fuzzy sets in decision-making systems has become a field of great interest, since Zadeh and Bellman introduced the fundamental elements about decision-making under fuzziness [5]. A decision in a fuzzy environment was defined as the intersection between fuzzy constraints and a fuzzy goal. Thus, if there is a fuzzy goal, G, and a fuzzy constraint, $C$, in a space of alternatives $X$, then $G$ and $C$ combine to form a decision, $\mathrm{D}$ :

$$
D=G \cap C
$$

When FRGS is extended to fuzzy decision-making, the knowledge about the goal (constraint) linked with the external input is included into the decision-making tasks. Thus, if the

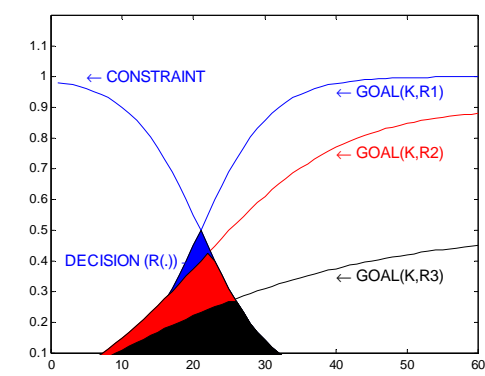

Fig. 3 - Adaptive fuzzy decision-making (different functions for goals).

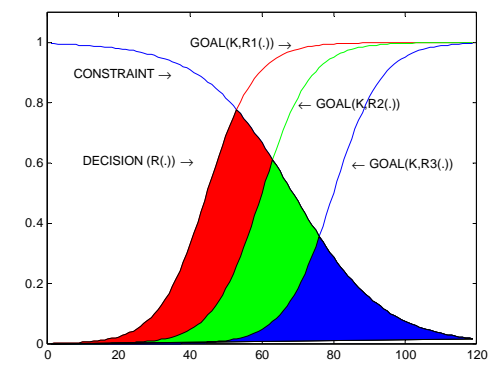

Fig. 4 - Adaptive fuzzy decision-making (same function, different parameters). goal changes, decisions need also to be modified from these external sources, as it follows:

$$
D=G[K(r)] \cap C[K(r)] .
$$

The knowledge, $K$, built into the set of rules, is altered, as there is changes in the reference, $r$. In this way, the reference is part of the goal (constraint) and is associated with the external sources present during the decision-making process.

Thus, in the same way that the reference composes the goal (constraint) that modifies membership functions in FRGS approach, the external factor will also alter membership functions in decision-making process. Fig. 3 and Fig. 4 depicted the ability of the FRGS approach to adapt the membership functions and thus deciding in an adaptive manner, when an external fact (goal) changes.

\section{FRGS Approach as Intelligent Agents: FRGS Agent}

Since the FRGS approach is itself a fuzzy control, it presents an equivalent behavior of intelligent agent as it has been adopted in this paper. However, because of the advantage of the FRGS control or decision-making process to adapt as function of the goal, it gives the ability to an agent to change its behavior in an adaptive manner. This approach gives a framework for an adaptive agent. Thus, if the FRGS approach is employed, fuzzy systems can not only be classified as reflexive agents with internal state, but also they are a kind of adaptive goal-based agent

Further, the FRGS approach is governed by the goal almost in the same way that the classical goal-based agent. Therefore, because the FRGS method changes its behavior (reasoning) to reach a desired task in accordance with the goal, this approach may also be sorted in the class of goalbased agent [16]. The only difference between them is that while in the latter one the goal govern directly the action to be used to change the environment, in the procedure proposed here the goal acts first upon the condition rules and membership functions, and then upon the environment. Thus, the FRGS approach is a kind of adaptive goal-based reflexive agent. It is a framework for adaptive reference-driven agent or, simply, a FRGS agent.

The FRGS agent is depicted in Fig. 5. The figure was built in accordance with the reflex agent and goal-based agent presented in [16]. This new framework for intelligent agents incorporates the expertise of humans; allows the imitation of human reasoning by using approximate reasoning; and adapts its behavior according to changes in the goal, as well as desire, intention, or belief if they are faced as exogenous inputs or parameters.

The FRGS agent supplies flexibility in decision and control action because it makes available adaptive actions to accomplish the agent's objective, even if it changes with time. 


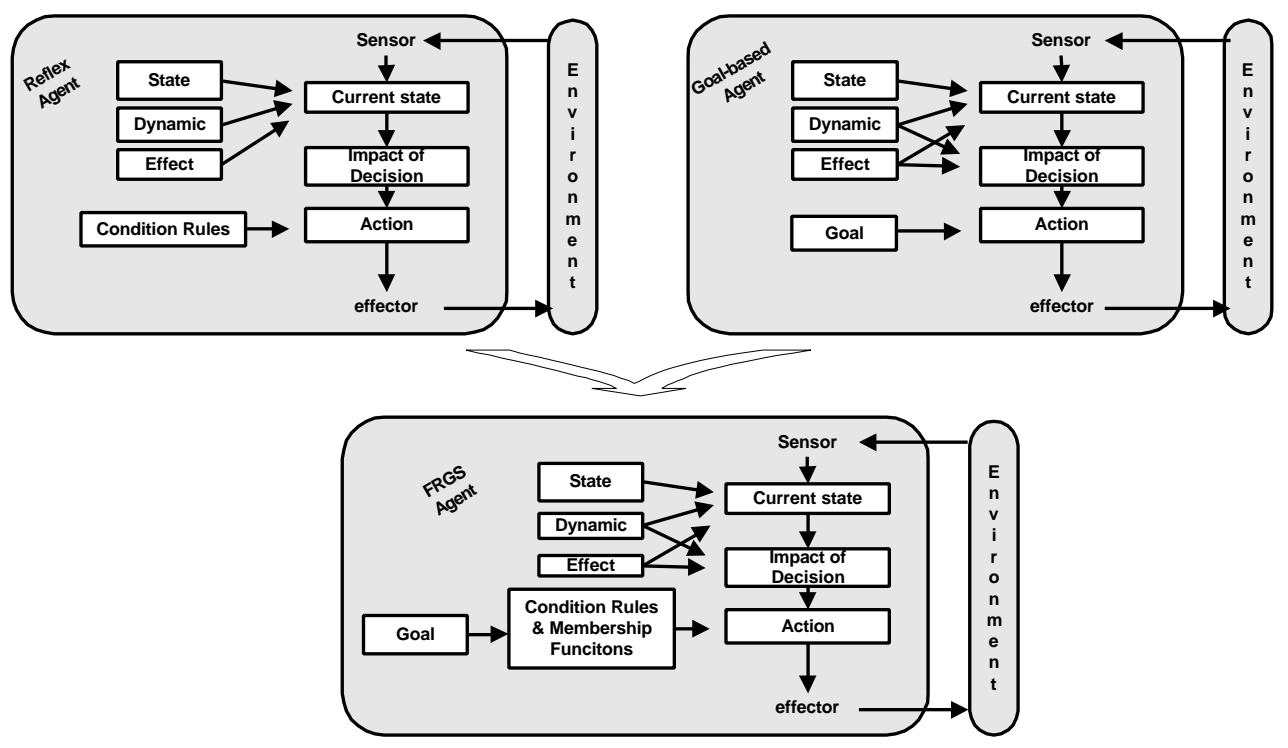

Fig. 5 - FRGS Agent

This flexibility allows the FRGS technique to reason about the problem by considering the future actions to be performed.

\section{FRGS Agent: A representative example}

When applied in control or decision-making problems the FRGS agent seems to allow emulating the adaptive human thinking. In this case, an intelligent agent would be designed to substitute a human in a control or decision-making process mainly if it is dealing with complex environment (systems). The conditional syllogism embedded in the human reasoning described by the method proposed here may be exemplified as it follows:

$$
\begin{array}{ll}
\text { If error }=\text { Small } & \text { THEN } \mathrm{u}=\text { Small; } \\
\text { If error }=\text { Medium } & \text { THEN } \mathrm{u}=\text { Medium; } \\
\text { If error }=\text { Large } & \text { THEN } \mathrm{u}=\text { Large },
\end{array}
$$

This set of IF-THEN rules represents the knowledge or expertise acquired by any individual. This representation is depicted in both Fig. 1 and Fig. 2. Although very similar in appearance, graphics (1a) and (2a) reveal a tiny difference introduced by small changes in the core and support of the membership functions. As proposed in this paper, these changes are obtained when exogenous inputs or parameters supply additional information related to new goals to be achieved (for instance, $r$ ).

Graphics (1b) and (2b) represent a perception of the environment (or measurement), by whichever device, related to information which will define the actual behavior of the system and that will affect the agent's final decision. The emulation of the human thinking performed by the agent is accomplished in sequence by the graphics (1c) and (2c), (1d) and (2d), and (1e) and (2e) for each example given. They respectively correspond to the cylindrical extension applied to the perception (measurement) over the knowledge base, conjunction principle, and projection principle. Finally, the areas in the graphics (f) reflect the resultant projection of the compositional rule of inference concerned with the final decision of the intelligent agent. Those areas are different since there are modifications of the membership functions that embody the knowledge base. Because the membership functions change considering the goal in this case, the final decision that determines the behavior of the agent over the environment is an adaptive goal-based decision. In so doing, this sort of decision fits the structure proposed in this paper for an adaptive goal-based agent called here as FRGS agent.

\section{CONCLUSION}

An emerging adaptive and goal-driven based on fuzzy reference gain-scheduling (FRGS) approach is presented in this paper. This kind of goal-based reflexive agent seems to be able to mimic the paradigms and mechanisms related to adaptive human decisions.

The FRGS methodology introduces adaptive control performance into autonomous systems, when there are changes in the objective (reference) to be achieved. The use of this concept in fuzzy decision-making process demonstrated its potential to cope with complex problem. Extended to intelligent agents this methodology becomes a FRGS agent that permits adaptive behavior according to goals, intentions, desires, or beliefs.

It is shown that the FRGS agent incorporates the main 
characteristics found in traditional intelligent agents. The proposed architecture for the agent is pro-active, autonomous, and reactive and, besides, it may present social ability.

Finally, the FRGS agent is an intelligent system able to emulate the human behavior by adapting the decision-making process according to the goal to be achieved in environments that present uncertainty, in the presence of vagueness, and/or when there is imprecise information.

\section{ACKNOWLEDGEMENT}

J.E.A.F. acknowledges support from the Brazilian research funding agency $(\mathrm{CNPq})$ under grant n. 381.212/97 and the Brazilian National Space Research Institute.

\section{REFERENCES}

[1] R.A. Aliev, B. Fazlollahi, R.M., Vahidov, "Soft Computing based multi-agent marketing decision support system", Journal of Intelligent Fuzzy Systems, v.9, n.1-2, pp.1-9, 2000.

[2] J.E. Araujo Filho, K.H. Kienitz, "Adaptive referencedriven decision-making process", Proc Intern. Conf. on Fuzzy Systems (FUZZ-IEEE),v.1, pp.452-457, St. Louis, 2003.

[3] J.E. Araujo Filho, S.A. Sandri, E.E.N. Macau, "A New Class of Adaptive Fuzzy Control System applied in Industrial Thermal Vacuum Process", Proc 8th IEEE Intern. Conf. on Emerging Technologies and Factory Automation (ETFA-IEEE), France, 2001, v.1, pp. 426431.

[4] J.E. Araujo Filho, S.A . Sandri, E.E.N. Macau, "Fuzzy Gain Scheduling Control Systems", In: Proc.: The 19th Intern. Meeting of the North American Fuzzy Information Processing Society (NAFIPS), Atlanta, EUA, July, 2000, pp. 461-464.

[5] R.E. Bellman, L.A. Zadeh, "Decision Making in fuzzy environment", Management Science, 1970, v. 17, pp. B141-B264.

[6] A. Bonarini, F. Basso, "Learning to compose fuzzy behavior for autonomous agents", Intern. Journal of Applied Reasoning, 1997, v. 17, pp. 409-432.

[7] M.E. Bratman, D.J. Israel, M.E. Pollack, "Plans and resource-bounded practical reasoning", Computational Intelligence, 1998, v.4, pp. 349-355.
[8] C.-H. Chang, Y. Chen, "Autonomous Intelligent Agent and its Potential Applications", Computers Ind. Engng. , 1996, v.31, n.1-2, pp.409-412.

[9] C.W. de Silva, Intelligent Control: Fuzzy Logic Applications, CRC Press Inc., 1995.

[10] C.W. de Silva, "Intelligent control of robotic systems with application in industrial processes", Robotics and Autonomous Systems, v.21, n.3, pp. 221-237, 1997.

[11] M.S. El-Nasr, J.Yen, R.R. Ioerger, "FLAME - Fuzzy logic adaptive model of emotions", Automatic Agent and Multiagent, v.3, n.3, pp.219-257, 2000.

[12] B. Fazlollahi, R.M. Vahidov, R.A. Aliev, "Multi-agent Distributed Intelligent System based on Fuzzy-Decision Making", Intern. Journal of Intelligent System, 2000, v.15, n.9, pp. 383-398.

[13] A.F. Gomez-Skarmeta, H. Martinez Barbera, "Fuzzy logic based intelligent agents for reactive navigation in autonomous systems", In Proc. Fifth Intern. Conf. on Fuzzy Theory and Technology, Raleigh, 1997, pp. 125131.

[14] S. Guillaume, "Designing fuzzy inference systems form data: An interpretability-oriented review", IEEE Trans. Fuzzy Systems, 2001, v.9, n.3, pp.426-443.

[15] P.McCauley-Bell, "Intelligent agent characterization and uncertainty management with fuzzy set theory: a tool to support early supplier integration", Journal of Intelligent Manufacturing, v.10, n.2, pp.135-147, 1999.

[16] S. Russel, P. Norvig, Artificial Intelligence- A Modern Approach, New Jersey: Prentice Hall Ed., 1995.

[17] M. Tarrazo, L.Gutierrez, "Economic expectations, fuzzy sets and financial planning", European Journal of Operational Research, v.126, n.1, pp.89-105, 2000.

[18] A. Tuma, H.J. Muller, "Using Fuzzy-directed Agents for Ecological Production Control", Intelligent Automation and Soft Computing, 2000, v.6, n.3, pp. 233-242.

[19]R.R. Yager, "Penalizing Strategic Preference Manipulation in Multi-agent Decision Making", IEEE Trans. Fuzzy Systems, 2001, v.9, n.3, pp. 393-403.

[20] R.R. Yager, "Fuzzy Sets and Approximate Reasoning in Decision and Control", Proc. IEEE, v.9, n.3, 2001.

[21] W.R. Zhang, "Nesting, safety, layering and autonomy: A reorganizable multiagent cerebellar architecture for intelligent control with application in legged robot locomotion and gymnastics", IEEE Trans. Systems, Man and Cybernetics - Part C, 1998, v.28, n.3, pp. 357-375.

[22]Zimmermann, H.-J., "Using fuzzy sets in operational research”, EJOR, 1987, v.13, pp. 201-216. 